\title{
Comprehensive Genome Engineering Toolbox for Microalgae Nannochloropsis oceanica Based on CRISPR-Cas Systems
}

\author{
Mihris Ibnu Saleem Naduthodi,* Christian Südfeld, Emmanouil Klimis Avitzigiannis, Nicola Trevisan, \\ Eduard van Lith, Javier Alcaide Sancho, Sarah D’Adamo, Maria Barbosa, and John van der Oost*
}

Cite This: ACS Synth. Biol. 2021, 10, 3369-3378

Read Online

ACCESS I

џll Metrics \& More

Article Recommendations

Supporting Information

ABSTRACT: Microalgae can produce industrially relevant metabolites using atmospheric $\mathrm{CO}_{2}$ and sunlight as carbon and energy sources, respectively. Developing molecular tools for highthroughput genome engineering could accelerate the generation of tailored strains with improved traits. To this end, we developed a genome editing strategy based on Cas12a ribonucleoproteins (RNPs) and homology-directed repair (HDR) to generate scarless and markerless mutants of the microalga Nannochloropsis oceanica. We also developed an episomal plasmid-based Cas12a system for efficiently introducing indels at the target site. Additionally, we exploited the ability of Cas12a to process an associated CRISPR array to perform multiplexed genome engineering. We efficiently targeted three sites in the host genome in a single transformation,

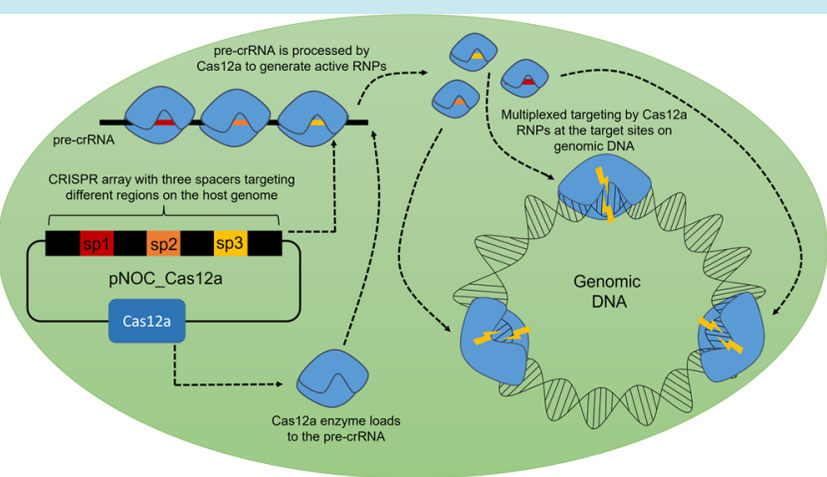
thereby making a major step toward high-throughput genome engineering in microalgae. Furthermore, a CRISPR interference (CRISPRi) tool based on Cas9 and Cas12a was developed for effective downregulation of target genes. We observed up to 85\% reduction in the transcript levels upon performing CRISPRi with dCas9 in N. oceanica. Overall, these developments substantially accelerate genome engineering efforts in $N$. oceanica and potentially provide a general toolbox for improving other microalgal strains. KEYWORDS: Nannochloropsis, microalgae, genome editing, CRISPR-Cas, gene silencing, ribonucleoproteins, Cas9, Cas12a

\section{INTRODUCTION}

The concept of microbial biofuel production has received major attention since it was realized that the exploitation of fossil fuels has become a threat to life on earth. Microalgae were already identified as a promising platform for sustainable production of biofuels in the 1950s. ${ }^{1-4}$ The possibility to achieve higher photosynthetic efficiency in microalgae (10\%) compared to that of land plants $(\sim 5 \%)$ and algal lipid accumulation reaching up to $60 \%$ of their cell dry weight (CDW) on the other hand makes them suitable candidates for biofuel production. ${ }^{5-10}$ Microalgae belonging to the genus Nannochloropsis have attracted scientific and industrial attention in the last decade, as they can accumulate lipids up to $60 \%$ of CDW under certain conditions, including high-value omega-3-polyunsaturated fatty acids (PUFA). ${ }^{11,12}$

Developing convenient genome editing tools is key for elucidating relevant details of the microalgal metabolism and for developing targeted variants with desired traits. CRISPR-Cas (clustered regularly interspaced short palindromic repeatsCRISPR-associated proteins)-based genome editing has been effectively implemented in Nannochloropsis spp. ${ }^{13-18}$ A type II Cas endonuclease from Streptococcus pyogenes named SpCas9 is the predominantly used Cas variant for nonhomologous end joining (NHEJ)-mediated generation of gene disruption mutants. ${ }^{19,20}$ To this end, the Cas9 gene was either integrated into the host genome or an episomal plasmid. The advantage of an episomal system is the possibility of generating nontransgenic mutants, as markerless deletions can be made, and the plasmid is generally cured upon relieve of antibiotic pressure. ${ }^{16,18}$ However, the dependence on ribozyme systems for precise sgRNA generation for Cas9-based editing ${ }^{21}$ required relatively complex and laborious cloning procedures to develop the plasmids for this approach.

Using the delivery of Cas ribonucleoproteins (RNPs) as an alternative to plasmid transfection could overcome the limitations posed by the plasmid-based systems. Almost a decade ago, homologous recombination (HR)-based insertion of an antibiotic resistance gene was reported as a tool for precise gene insertion or targeted gene disruption in Nannochloropsis spp. ${ }^{22}$ In our previous study, we improved the HR efficiency in Nannochloropsis oceanica using Cas RNPs for generating double

Received: July 15, 2021

Published: November 18, 2021 
A

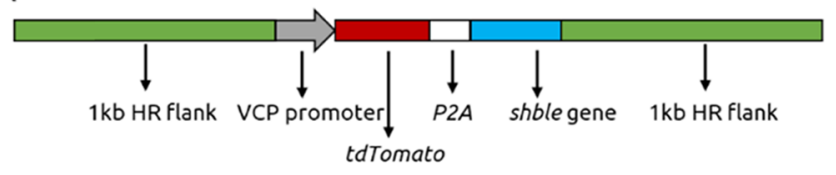

C

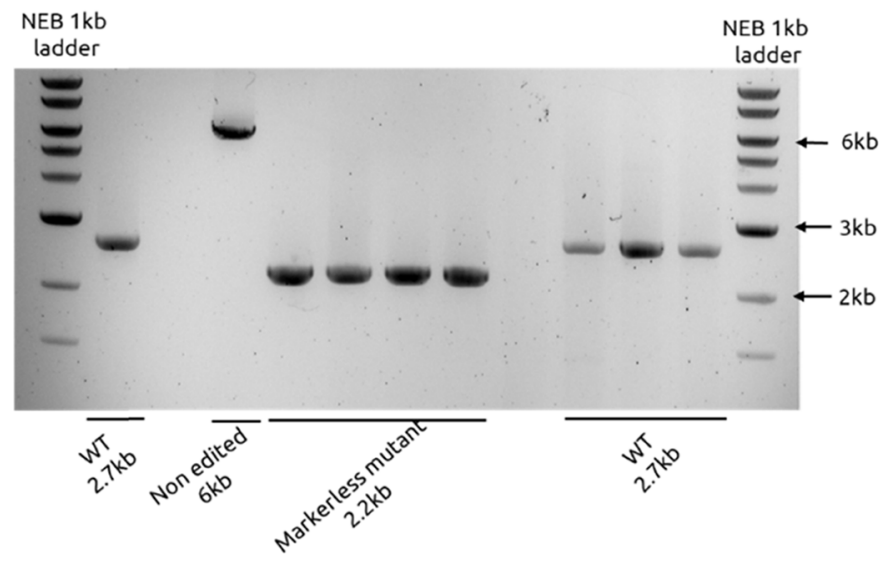

B

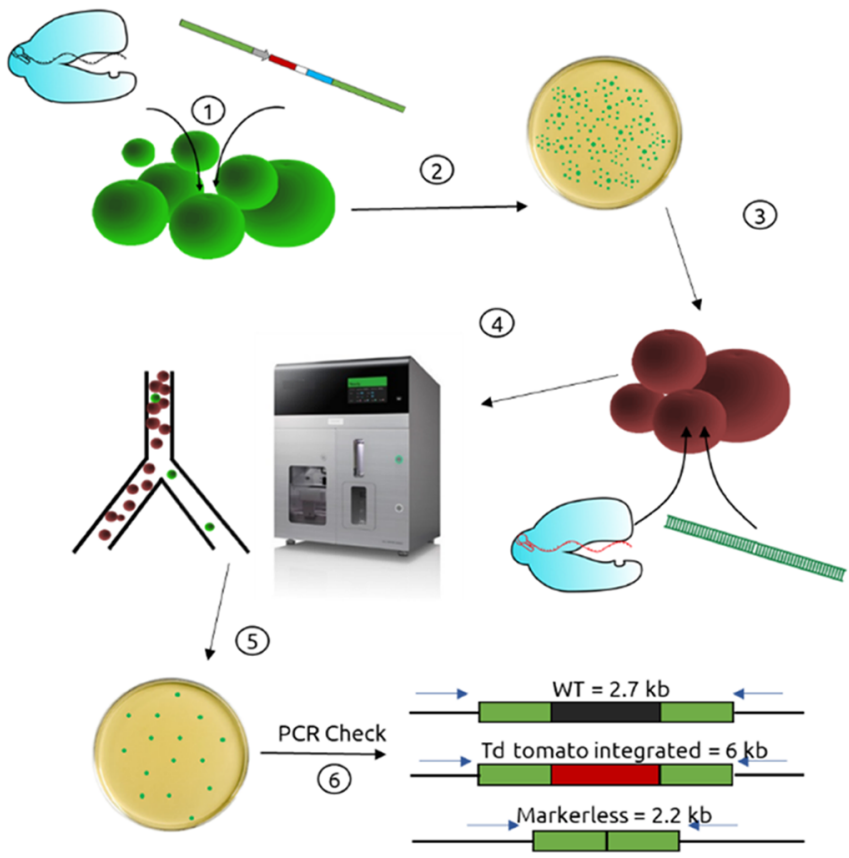

Figure 1. RNP-based genome editing strategy for generating markerless mutants. (A) Editing template for introducing the tdTomato fluorescence gene. (B) RNP-based approach for generation of scarless mutants: (1) transformation of the editing plasmid and RNP targeting the nitrate reductase gene, (2) selection on antibiotic plates, (3) selection and scaling up of mutants harboring designed integration of the editing plasmid and tdTomato expression, (4) transformation of tdTomato-expressing mutants with the editing plasmid and RNP targeting the tdTomato gene, (5) sorting of cells that lack the tdTomato expression and growing on solid media without antibiotic selection, and (6) PCR on obtained colonies to identify the scarless and markerless mutants. (C) PCR analysis showing the various genotypes obtained after the sorting.

strand breaks (DSBs) at the DNA target site that were subsequently restored by the host's homology directed repair (HDR) system. ${ }^{17}$ However, the mutants developed by this approach did harbor an antibiotic resistance cassette at the target site. This outcome is a drawback for generating multiple knockouts as the available antibiotic resistance genes are limited for Nannochloropsis spp. ${ }^{23}$

Here, we describe further development of the RNP-based strategy for generating scarless mutants in N. oceanica using fluorescence-activated cell sorting (FACS). Additionally, we now developed a plasmid-based genome editing system employing Francisella novicida Cas12a (FnCas12a). ${ }^{24,25}$ As such, we did overcome the dependence on ribozyme-based guide maturation systems by exploiting the ability of Cas12a to autoprocess the precursor CRISPR RNA (pre-crRNA) to mature crRNA guides, as a way to simplify the plasmid development. The latter feature of Cas12a also allowed us to develop a multiplexed targeting system that can simultaneously produce multiple knockouts in $N$. oceanica in a single transformation. Moreover, we also report an effective CRISPR interference (CRISPRi) tool for downregulating target gene expression using Cas9 and Cas12a proteins.

\section{RESULTS AND DISCUSSION}

Lb/FnCas12a RNP-Based Markerless Mutant Generation and FACS-Based Selection. Developing nontransgenic mutants using RNPs can have widespread applications in microalgal biotechnology. To this end, we set out to utilize the phenotypic variation of transformants expressing a fluorescence marker integrated at the target site followed by a FACS- mediated negative selection to generate markerless microalgal mutants.

The first step toward exploiting FACS for markerless genome editing was to knock-out the target gene by replacing it with a fluorescence marker gene. To this end, the zeocin resistance (ZeoR) gene (shble, GenBank accession number A31898.1) described previously ${ }^{17}$ was linked, via the 30 amino acid long P2A peptide sequence to the gene encoding the DsRed variant tdTomato $^{26,27}$ (Figure 1A). This selection marker construct was inserted between HR flanks designed to precisely knock-out the nitrate reductase (NR) gene. The NR gene was selected as the target because characterizing $N R$ mutants is easily achieved by testing for their loss of ability to grow on nitrate. ${ }^{13,16}$ The RNP used for transformation was composed of a sgRNA targeting the $N R$ gene and the Lachnospiraceae bacterium Cas12a (LbCas12a) protein. After transformation of the RNP and the linear marker fragment, obtained colonies were screened by PCR to check for predicted insertion of the editing template at the target site. Among 19 colonies, two colonies showed the desired target gene substitution. The mutant strains were analyzed by flow cytometry to confirm tdTomato expression. The fluorescence emission of mutants was easily separable from the wild-type cells (Figure S1).

In the second step, we aimed to remove the ZeoR-tdTomato marker cassette to produce a markerless mutant. To this end, we generated two RNP complexes, consisting of F. novicida Cas12a (FnCas12a) and two crRNAs for (in parallel) targeting different positions of the tdTomato gene. In combination with a new linear editing template only consisting of the previously used 1 Kb HR flanks joined together (Figure 1B), the ZeoR/tdTomatoexpressing parental strain was transformed with the new RNPs. 
The transformed cells were recovered for 6 days to allow for degradation of the tdTomato protein. Next, the recovered transformants that contained a mix of nonedited parental (high tdTomato fluorescence) and edited (low tdTomato fluorescence) were sorted by FACS (Figures $1 \mathrm{~A}$ and S1). From two independent transformations for each RNP, cells were sorted by FACS to obtain tdTomato-free clones that were screened by colony PCR: 87 colonies for RNP with spacer 1 and 91 colonies for RNP with spacer 2 . We observed that all of the colonies obtained via RNP with spacer 1 were false positives and did not contain any markerless mutants. However, for RNP harboring spacer 2, 4/91 colonies showed the expected band size of markerless mutants (Figure 1C). Sequencing of PCR products confirmed the complete deletion of the tdTomato and Zeocin resistance cassette from the host genome, thus indicating that markerless nitrate reductase gene knockouts were successfully obtained.

CRISPR-Cas-based genome editing in microalgae was first reported with feeble efficiencies in Chlamydomonas reinhardtii in 2014. ${ }^{28}$ Since then, various strategies were implemented in multiple microalgal strains to improve editing efficiencies or to perform compelling genetic engineering studies. ${ }^{29,30}$ However, in most studies, the Cas 9 gene was incorporated into the host genome, resulting in transgenic mutants. ${ }^{13-15,31-33}$ RNP-based approaches circumvented this bottleneck and developed markerless mutants by disrupting the target site with indels. ${ }^{17,34-37}$ This approach was initially demonstrated in C. reinhardtii to generate markerless knockouts by introducing indels in the CDS (coding DNA sequence) of the target gene. $^{34,36}$ The selection of mutants in these studies was facilitated by the difference in the color of the mutant colonies or auxotrophy after transformation. Thus, generating mutants that do not produce a selectable phenotype can be cumbersome via this approach. Introducing an antibiotic resistance cassette at the target site by Cas RNP-mediated HDR followed by selection on antibiotic media and subsequent screening for mutants by PCR addressed this bottleneck. ${ }^{17}$ However, the presence of an antibiotic resistance cassette in the mutants developed by this approach restricted further engineering due to limited antibiotic resistance genes available for microalgae. Also, commercialization of mutant strains that harbor non-native genes is restricted in some countries. ${ }^{38}$ Thus, developing nontransgenic mutants can have multiple advantages from a scientific, industrial, and societal perspective. In this study, for the first time, we provide a strategy to develop nontransgenic mutants using RNPs and HDR in N. oceanica. Future efforts could focus on optimizations in this method to further improve the efficiency in obtaining the markerless mutant colonies. The presence of false positives in the sorted colonies has been a bottleneck of the approach and can be reduced by optimizing the selection gate of colonies during the FACS. Also, the sorting can be performed on different days after the second round of transformation to identify the optimal recovery time that can yield maximum efficiency.

Codon Harmonization and Expression of $f$ Cas $12 a$ in N. oceanica. The RNP-based approach described above required two rounds of transformation and selection via FACS to develop markerless mutants. Moreover, the high cost of purified Cas protein and the requirement of screening via FACS could make this approach inaccessible to wider microalgal researchers. Thus, to further accelerate the generation of mutants and to simplify the screening process, we set out to develop an episomal plasmid-based Cas12a system for genome editing in N. oceanica. We adapted a recently developed episomal system for $N$. oceanica to develop our Cas12a-based genome editing system. ${ }^{16}$ This system harbors a Cas9 gene sequence and ribozyme-mediated production of guide RNAs and it was used for deleting a $100 \mathrm{~kb}$ fragment in $N$. oceanica. ${ }^{18}$ However, using ribozymes for precise generation of guide RNAs complicates plasmid production (especially in the case of multiplexed genome editing) and limits the usefulness of this system for high-throughput applications. Developing a Cas12a episomal plasmid system based on this chassis could expand the genetic toolbox for Nannochloropsis and address the bottlenecks associated with the Cas9 system. Moreover, in vivo studies in human cells have shown that the off-target effects of the Cas12a enzyme are comparatively lower than Cas $9 .{ }^{39}$ We opted to use the FnCas12a variant due to its improved activity in $N$. oceanica compared to LbCas12a and AsCas12a. ${ }^{17}$

The $f_{n}$ Cas $12 a$ gene sequence was codon-harmonized for the expression in $N$. oceanica using the online Galaxy codon harmonizer tool. ${ }^{40}$ To the $3^{\prime}$ end of the harmonized $f_{n}$ Cas $12 a$ gene, an in-frame fusion was made with a gene encoding the reporter protein Nano Luciferase (Nlux) to allow validation of Cas12a expression in transformants. ${ }^{16,41}$ Expression of the fusion protein was regulated by the bidirectional Ribi promoter in the resulting plasmid pNOC_nfnCas12a-NLux. Wild-type $N$. oceanica was transformed with this plasmid and transformants were selected for antibiotic resistance. Colonies were screened for Nlux activity, but no luminescence was detected in any of the colonies.

Human codon-optimized Cas9 was previously shown to be effective in N. oceanica. ${ }^{16}$ Assuming that the codon-harmonized $f_{n} \operatorname{Cas} 12 a$ gene sequence was the system's bottleneck, we expressed human codon-optimized $f_{n} \mathrm{Cas} 12 a$ and $l b C a s 12 a$ to identify the best-expressed Cas gene sequences for $N$. oceanica. The plasmids pNOC_hfnCas12a-Nlux (encoding the humanized $f_{n}$ Cas $12 a-N l u x$ fusion protein) and pNOC_hlbCas12aNlux (encoding the humanized $l b C a s 12 a-N l u x$ ) were transformed, selected using the ZeoR marker, and screened for Nlux activity. In this case, the Nlux activity was observed for both variants and the activity of the fnCas12a-Nlux fusion protein was higher than that of the lbCas12a-Nlux (Figure S2). This observation indicates that the codon harmonization can be detrimental for expression of certain genes and warrants further investigation. The rare codons present in the harmonized and human-optimized Cas12a sequences are presented in Supporting Information Figures S3-S5. ${ }^{42}$ The harmonized sequence indicates the presence of multiple rare codons, while the codons in the humanized Cas12a sequences are favorable for expression in Nannochloropsis. In line with this observation, humanized Cas $12 a$ sequences are found to be suitable for expression and genome editing in $N$. oceanica. The humanized Cas 9 gene sequence was also previously reported to be efficiently expressed in N. oceanica. ${ }^{16}$ The humanized $f_{n} C a s 12 a$ gene was selected for the next round of experiments owing to its improved expression and previously validated high activity in N. oceanica.

Episomal fnCas12a System Introduces Indels at the Target Site. Next, we set out to use the humanized $f n C a s 12 a$ as a genome editing tool for $N$. oceanica by generating indels at a target site. To this end, along with the $f_{n} \operatorname{Cas} 12 a$, we used a fulllength CRISPR RNA (crRNA) with a 36 nucleotide (nt) direct repeat (DR) followed by a $25 \mathrm{nt}$ long spacer sequence targeting the gene of interest. We selected three spacer sequences targeting the exon of the NR gene (crRNA NR1, crRNA NR2, and crRNA NR3). The bidirectional Ribi promoter regulated the transcription of Cas12a and the crRNA (Figure 2A). To 


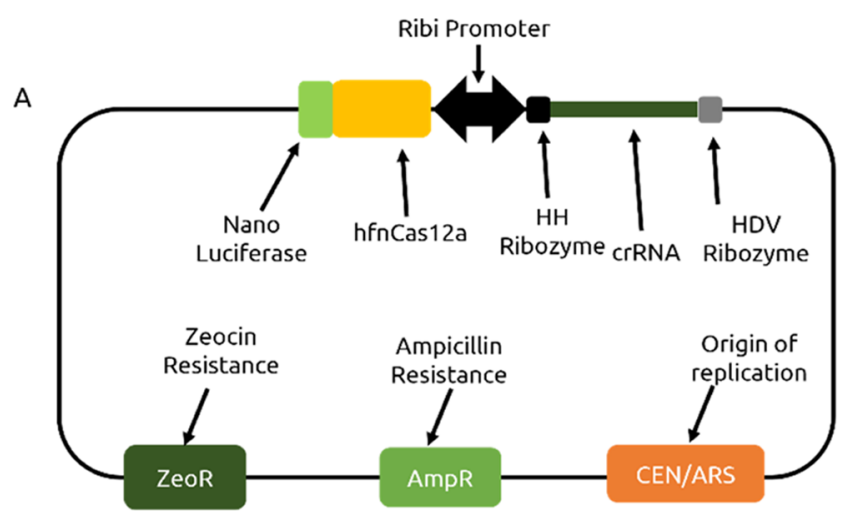

C

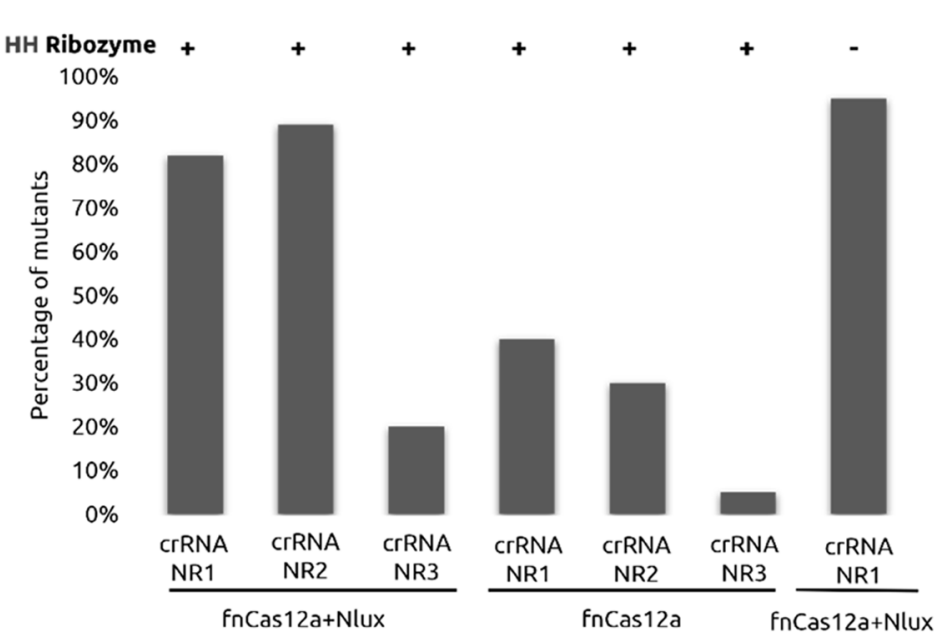

B

CIRNA NR1:

GTCTAAGAACTTTAAATAATTTCTACTGTTGTAGATCCACTTCCACGACAATCGCGTCCTG

CIRNA NR2:

GTCTAAGAACTTTAAATAATTTCTACTGTTGTAGATTGGCACGAGTATCCCCCTCCTCAAG

CrRNA NR3:

GTCTAAGAACTTTAAATAATTTCTACTGTTGTAGATCAGTCTCGTCGGCCCCTGCCTCCAT

Repeat sequence 36 nts

Spacers $25 n$ ts

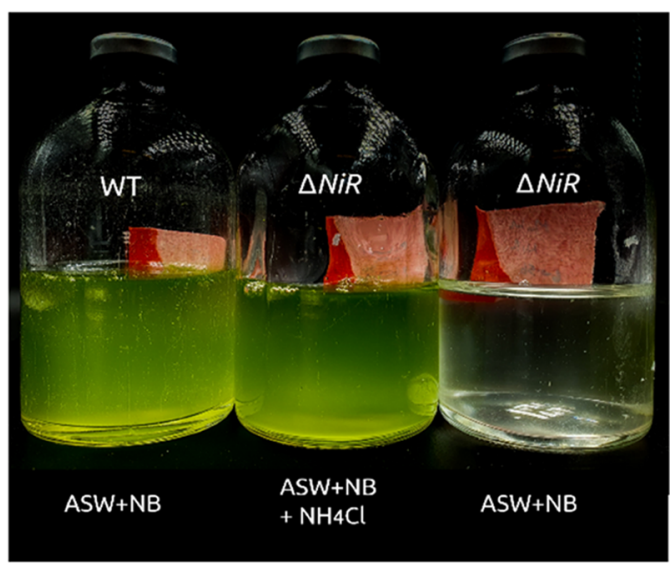

Figure 2. Plasmid-based Cas12a efficiently introduces DSB at target sites in N. oceanica. (A) Schematic map of the episomal plasmid system used for generating NHEJ-based indel mutations in N. oceanica. (B) Various CRISPR RNA sequences targeting different positions of the nitrate reductase gene. (C) Efficiencies of obtaining indel mutants upon using various plasmids. (D) Phenotypic assay of nitrate reductase mutants shows the inability of mutants to grow in media without ammonia.

facilitate precise crRNA formation from mRNA molecules, we added the hammerhead $(\mathrm{HH})$ and hepatitis delta virus (HDV) ribozymes on either end of the crRNA sequences. ${ }^{16,43,44}$

The plasmids pNOC_hfnCas12a-Nlux_crRNA NR1/NR2 and NR3 were transformed into wild-type $N$. oceanica, and 96 colonies per plasmid were selected and screened for Nlux activity. Colonies (72, 55, and 75\%) from plasmids pNOC_hfnCas12a-Nlux_NR1/NR2 and NR3 showed Nlux activity. Screening of these colonies for indels at the target site by PCR and sequencing revealed that 82,89 , and $20 \%$ of the Nluxpositive colonies for plasmids harboring crRNA 1, 2, and 3, respectively, showed indels at the target sites and these colonies required ammonia to grow (Figures $2 \mathrm{C}, \mathrm{D}$ and S6-S8). When removing the Nlux tag from the $f_{n} \mathrm{Cas} 12 a$ gene and screening transformant colonies directly by PCR, indel formation efficiencies were 40, 30, and 5\% for pNOC_hfnCas12a_crRNA NR1, NR2, and NR3, respectively (Figure $\overline{2} \mathrm{C}$ ).

This one-step approach to generate markerless mutants is substantially simpler than the aforementioned two-step procedure using negative fluorescence selection with FACS. However, it cannot easily be used for knocking out multiple genes at the same time due to the complicated cloning of multiple $\mathrm{HH}$ ribozyme sequences. Therefore, we next tested whether the innate crRNA processing ability of Cas12a can be used to improve the plasmid-based gene knock-out system by simplifying the cloning procedure. To assess the crRNA processing capability of fnCas12a in N. oceanica, we removed the $\mathrm{HH}$ ribozyme from the plasmid $\mathrm{pNOC}$ hfnCas12a_crRNA NR1 to develop the plasmid pNOC_h̆hnCas12a_No_nu_($\mathrm{HH})$ crRNA NR1. We achieved an editing efficiency of $\overline{95} \%$ with this plasmid (Figure 2C), indicating effective selfprocessing of crRNA by the Cas12a enzyme in $N$. oceanica. This removal of the $\mathrm{HH}$ ribozyme further simplifies the introduction of spacers into Cas12a plasmids for genome editing in this microalga.

Multiplexed Genome Editing in N. oceanica Using fnCas 12a. The efficient processing of crRNA by Cas12a can be exploited for multiplexed genome editing in N. oceanica. ${ }^{25,45}$ To this end, we introduced a CRISPR array in the crRNA locus of the targeting plasmid pNOC_hfnCas12a_crRNA NR1. The CRISPR array consisted of three 36 nt DR sequences interspaced by the spacer sequences NR1 and NR2 (Figure 3A). This plasmid was developed by the CRATES assembly, a simplified method for generating CRISPR arrays for multiplexed genome engineering. ${ }^{46}$ The NR1 and NR2 target sites were 375 nucleotides apart in the NR gene. Simultaneous targeting at both loci could either result in deletion of the region between the targets or introduce indels at both target sites. We transformed wild-type $N$. oceanica with the multiplexing plasmid pNOC_hfnCas12a_Nlux NR1_NR2 and selected colonies with Nlux activity for screening by PCR. We observed a reduction (approx. 375 base pairs) in the amplicon size for 11/40 (27\%) colonies, 


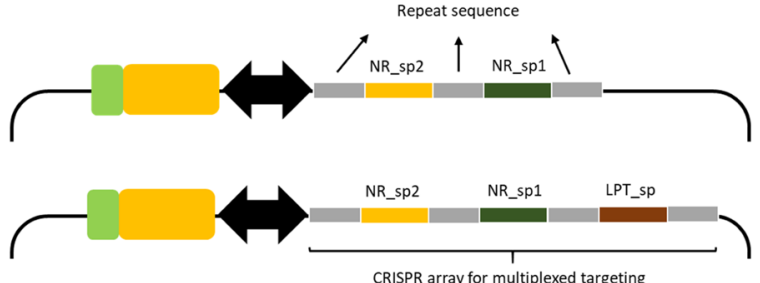

B

\section{GTCTAAGAACTTTAAATAATTTCTACTGTTGTAGATCCACTTCC ACGACAATCGCGTCCTGTGCTG GTCTAAGAACTTTAAATAATTTCTACTGTTGTAGATTGGCACGA GTATCCCCCTCCTCAAG \\ GTCTAAGAACTTTAAATAATTTCTACTGTTGTAGATCTTTGTCG CTCGACGTGTCCAAGGG \\ GTCTAAGAACTTTAAATAATTTCTACTGTTGTAGAT}

Figure 3. Multiplexing plasmids and the CRISPR array. (A) CRISPR array used for multiplexed genome engineering in $\mathrm{N}$. oceanica. The spacers targeting the nitrate reductase gene are indicated in yellow and green. The dark red colored spacer targets the LPAAT gene and was used for multiplexed editing at 3 loci in a single transformation. (B) CRISPR array sequence of the multiplexing plasmid targeting 3 regions. The colors used for the nucleotides correspond to the annotation in panel (A). The 5 nucleotides depicted in red (TGCTG) are the extensions used for the CRATES assembly to develop the plasmid.

indicating efficient multiplexed targeting and deletion of the region between the target sites (Figure S9). Sequencing of PCR products confirmed this (Figures S10 and S11) and showed that an additional 4 colonies had indels at both target loci, resulting in a total of $37 \%$ multiplexing efficiency. We further observed that 14/40 (35\%) colonies carried mutations at the NR2 site, whereas no mutants had indels only at the NR1 site.

To further expand the multiplexing potential, we added an additional spacer to the CRISPR array to target three regions in a single transformation ( $\mathrm{pNOC}$ hfnCas $12 \mathrm{a}+\mathrm{N}$ lux_NR1_NR2_LPT1) (Figure 3B). The additional spacer targeted the $L P \bar{A} T 1$ gene, as successful nonlethal knock-out of this gene has been demonstrated in $N$. oceanica previously. ${ }^{47}$ After transformation and selecting 48 Nlux-positive colonies, we obtained 7/50 (14\%) colonies that harbored mutations at all 3 target sites. Colonies $(2 / 50,4 \%)$ showed different combinations with mutation at two target sites, while $6 / 50(13 \%)$ colonies had a mutation at either one of the target sites. These results indicate the potential of using Cas12a for generating multiplexed knockout mutants in microalgae. The mother plasmid we developed (pNOC_hfnCas12a_Nlux_CRATES) harbors type $2 \mathrm{~S}$ restriction sites at the CRISPR array locus, which can be used for swift generation of multiplexing constructs, either by CRATES or Gibson assembly.

The dispensability of ribozymes and successful multiplexed targeting could make Cas12a the preferred Cas variant over Cas9 for genome editing in microalgae. Nevertheless, the multiplexed targeting efficiencies were lower compared to single targeting, as observed in other microorganisms. ${ }^{45}$ The secondary structures formed by long CRISPR arrays could complicate the processing by Cas12a to form active crRNAs and this could be a

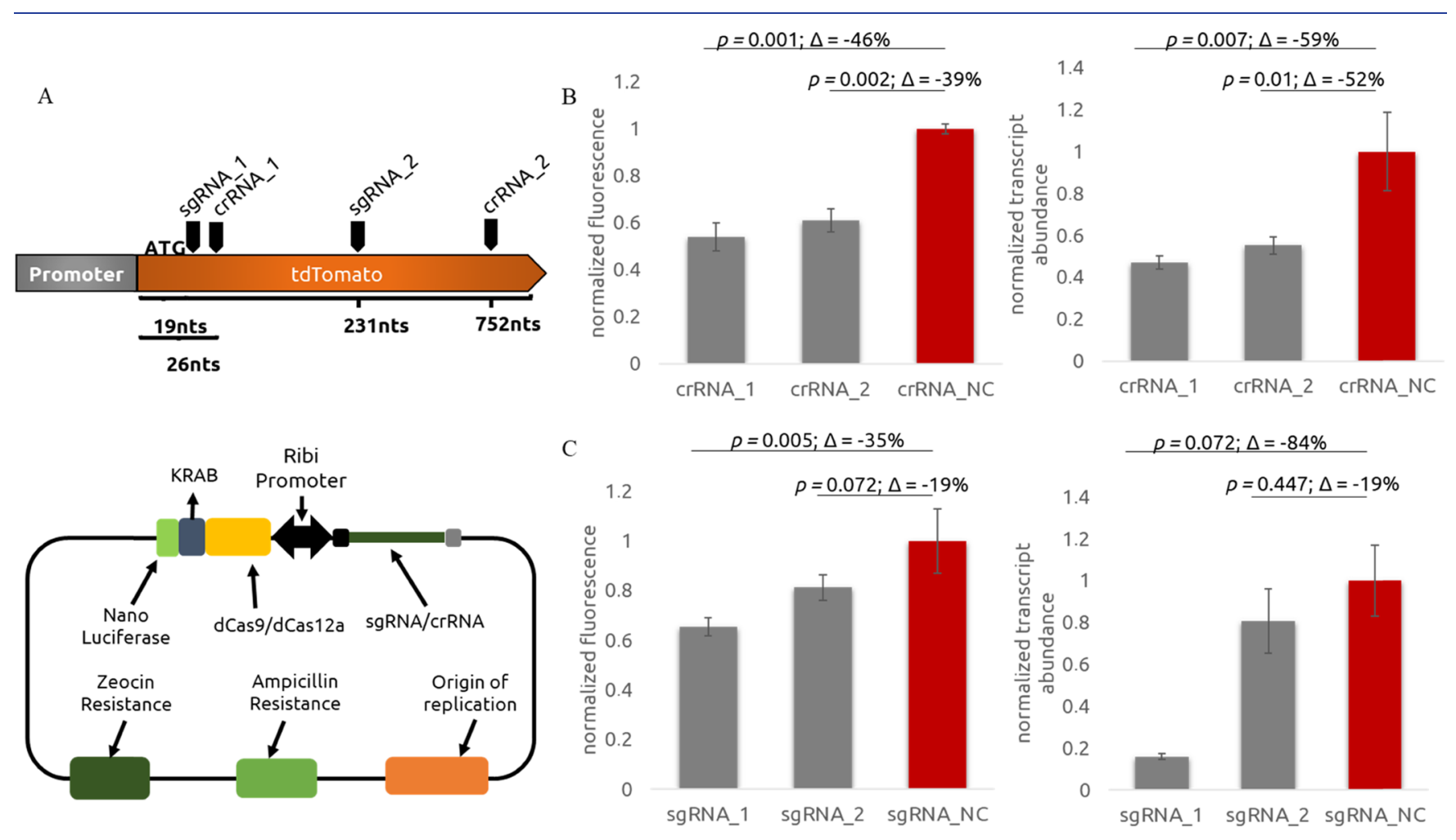

Figure 4. Silencing by CRISPR interference (CRISPRi). (A) Map of dCas-KRAB plasmids for CRISPRi in N. oceanica and the distance of different target sites from the start codon in the CDS of tdTomato gene. (B) Fluorescence and mRNA levels (qPCR) of various dCas12a-KRAB plasmids targeting the tdTomato gene. (C) Fluorescence and mRNA levels (qPCR) of various dCas9-KRAB plasmids targeting the tdTomato gene. Fluorescence is normalized to OD and the fluorescence value of NC. Fluorescence (/transcript) values were normalized to the mean value of the negative control. Error bars denote the standard error of the mean $(N \geq 2)$. Group means were compared for a significant difference to the control using Tukey's HSD test. Relative differences compared to the control are given $(\Delta)$. 
major factor that contributes to reduced efficiencies. Switching the position of spacer sequences in the CRISPR array has shown to have varying effects on targeting efficiencies. ${ }^{48}$ This could be a potential approach to optimize the CRISPR arrays and improve the multiplexed targeting efficiencies. Moreover, using a truncated repeat sequence (19 nts) in place of the full-length 36 nts long repeats could reduce the complexity of the long CRISPR array transcript. CRISPR arrays with truncated repeat sequences in combination with AsCas 12a had higher efficiencies in generating indels in human cells. ${ }^{25}$ This could also be a potential strategy to enhance the multiplexed targeting efficiencies in $N$. oceanica. The maximum number of target sites that can be edited in a single transformation in N. oceanica can only be identified by developing long CRISPR arrays with a large number of spacers. In human cells, up to $25 \mathrm{crRNAs}$ were produced from a single CRISPR array. ${ }^{49}$ However, further optimizations as discussed above will be required in obtaining multiplexed mutants with indels at all of the target site as the number of spacers is increased on the CRISPR array.

CRISPRi-Mediated Gene Downregulation. Next to the aforementioned gene disruption approaches, the development of an efficient transcription repressor system would be very useful for accelerating fundamental studies in microalgae. The specific silencing of gene expression by CRISPRi has been successfully implemented in a wide range of organisms, including the microalga $C$. reinhardtii. ${ }^{50-53}$ A catalytically inactive Cas 9 tagged with a transcriptional repressor domain has shown $94 \%$ gene silencing efficiency in Chlamydomonas. ${ }^{53}$ We aimed to identify the efficiency of FnCas12a and SpCas9 in downregulating gene expression in N. oceanica. To this end, we developed catalytically inactive variants of SpCas9 by introducing two mutations D10A and $\mathrm{H} 840^{50}$ and catalytically inactive fnCas 12a by introducing mutations E1006A and D917A. ${ }^{24,54,55}$ The deadCas (dCas)-encoding genes were introduced in the aforementioned episomal system and replaced the ZeoR cassette with a blasticidin resistance cassette to establish the CRISPRi plasmids for N. oceanica.

Ideal target genes for this endeavor should facilitate simple quantification of gene expression. However, the wild-type $N$. oceanica did not harbor a gene that met our requirements. For that reason, we used a previously constructed host strain in which the gene encoding tdTomato was used to knock-out the NR-encoding gene (Figure 1A, first step). We developed dCas9 and dCas12a episomal plasmid systems with spacers targeting two regions of the tdTomato CDS. Plasmids expressing either Cas proteins together with a nontargeting spacer sequence were used as controls. After transformation of the tdTomatoexpressing $\left(\right.$ tdTomato $\left.^{+}\right)$parental strain, colonies were selected on solid media with ammonia and blasticidin. Three colonies with active Nlux expression per construct were grown in liquid media to the exponential phase and tdTomato fluorescence levels were quantified by a plate reader. However, none of our transformants exhibited a significant reduction in tdTomato fluorescence levels (Figure S12). This outcome indicates that the expression of dCas9 and dCas12a proteins might not be an effective CRISPRi tool for microalgae.

Attaching transcription repressor domains (TRD) to dCas proteins has been reported to enhance the CRISPRi efficiency in various organisms. ${ }^{56-60}$ Among various TRDs available, we selected Kruppel associated box (KRAB) for tagging dCas proteins as this variant domain was active in Chlamydomonas. ${ }^{53}$ Next, we developed the same plasmids as in the previous experiment but with the KRAB domain sequence attached to the
dCas-encoding sequences at their 3 '-termini (Figure 4A). After transformation, selection on the antibiotic, and Nlux screening, two or three Nlux-positive colonies per transformation were screened for tdTomato fluorescence during the exponential growth phase. Various tdTomato downregulation levels were observed for cultures harboring plasmids with spacers targeting the tdTomato gene (Figure 4B,C). Reduction in fluorescence levels was observed for both dCas9-KRAB and dCas12a-KRAB. To further validate this observation, RNA isolation followed by cDNA synthesis and qPCR was performed (Figure 4B,C), which revealed up to $85 \%$ reduction in transcript levels for spacers targeting the beginning of the CDS with dCas9-KRAB. The highest repression with dCas $12 \mathrm{a}-\mathrm{KRAB}$ was also observed for spacer targeting the 5 '-end of the CDS. Weaker repression was observed for spacers targeting loci further downstream the start codon of the target gene. These observations are in line with previous studies showing reduced CRISPRi upon targeting more toward the $3^{\prime}$-end of the CDS and higher efficiencies when targeting close to the transcription/translation start site. ${ }^{50,61}$ Moreover, targeting the promoter sequence is reported to provide strong silencing of the target gene in other organisms. ${ }^{50}$ This could also be a potential target to strongly downregulate the gene expression in microalgae.

Here, we report substantial improvements to the genome editing toolbox for microalgae that address most of the bottlenecks associated with the present CRISPR-Cas tools. Combining the low risk of off-target effects when using transiently present Cas RNP with the ability to generate scarless mutants could be exploited for the sequential generation of nontransgenic mutants with multiple alterations in the genome. However, further optimizations are required to improve the efficiencies of obtaining markerless mutants via FACS. The development of plasmid-based systems that employ Cas12a is a valuable addition to the existing Cas9 system, as it expands the available target sites in the A-T-rich regions of the host genome for microalgal genome editing. We developed a proof of concept that validates the application of Cas12a for multiplexing in $N$. oceanica. To this end, we demonstrated successful targeting at three loci in a single transformation and this could be further elaborated in future studies to edit more targets by elongating the CRISPR array with required spacers. Furthermore, efficient multiplexing and the elimination of the need for ribozymes compared to Cas9 systems strengthen the position of Cas12a as the enzyme of choice for genetic engineering of Nannochloropsis spp. and other microalgae. Finally, the development of an efficient CRISPRi system completes the essential tools required for extensive microalgal genome engineering. Expanding the genome editing toolbox with improved techniques will accelerate the development of microalgae variants for fundamental and applied purposes. ${ }^{62}$

\section{MATERIALS AND METHODS}

Strain and Media Composition. The N. oceanica IMET1 was kindly provided by Prof. Jian Xu from QIBET, Chinese Academy of Sciences. The strains were cultured in artificial seawater, as previously reported, ${ }^{17}$ and supplemented with commercially available "nutribloom plus" (ASW-NB) provided by Necton (Olháo, Portugal). The liquid microalgae were grown with $5 \% \mathrm{CO}_{2}$ and $3 \mathrm{~g} / \mathrm{L}$ sodium bicarbonate as the buffer to maintain the $\mathrm{pH}$ at 7.9-8.0. For maintenance, recovery after transformation, and selection on antibiotics, the cultures were grown in light incubators at $25{ }^{\circ} \mathrm{C}$ with a constant light intensity of $50 \mu \mathrm{mol} / \mathrm{m}^{2} / \mathrm{s}$. For growing cells without $\mathrm{CO}_{2}, 4.77 \mathrm{~g} / \mathrm{L}$ 
HEPES was added to the media and $\mathrm{pH}$ was set to 8 . For nitrate reductase knockouts, a final concentration of $12 \mathrm{mM}$ ammonium chloride was added to the ASW-NB media. Antibiotics zeocin $(10 \mu \mathrm{g} / \mathrm{mL})$ or blasticidin $(100 \mu \mathrm{g} / \mathrm{mL})$ were used to select and cultivate transformants.

Plasmid Construction. All of the plasmids in the study were assembled using Gibson assembly $(2 \times \mathrm{HiFi}$ DNA assembly master mix from New England Biolabs, NEB) with the exception of the multiplexing plasmid that was cloned by CRATES assembly (Supporting Information). ${ }^{46}$ The details on plasmid construction and the GenBank files of plasmids used in the study are provided in the Supporting Information. The Cas12a sequences obtained from plasmids pY004 (pcDNA3.1hFnCpf1) and pY016 (pcDNA3.1-hLbCpf1) were developed in the group of Feng Zhang (Addgene plasmid \#69976 and \#69988). ${ }^{24}$ The Cas9 sequence was a gift from Farre. ${ }^{16}$ The fragments required for the cloning procedures were amplified by PCR using Q5 High Fidelity $2 \times$ Master Mix (NEB), and the primers for PCR were obtained from Integrated DNA Technologies and their sequences are tabulated in Table S1.

Transformation and Selection. A previously described electroporation protocol ${ }^{63}$ was adapted for transforming the $N$. oceanica IMET 1 strains. The microalgal cells were inoculated to an $\mathrm{OD}_{750}$ of 0.08 and grown under medium light conditions $\left(\sim 50 \mu \mathrm{mol} / \mathrm{m}^{2} / \mathrm{s}\right)$ for 2 days. For each transformation, $50 \mathrm{~mL}$ of culture was harvested at the mid-exponential growth phase $\left(\mathrm{OD}_{750}\right.$ of 0.5$)$ by centrifugation $\left(2000 \mathrm{~g}, 10 \mathrm{~min}\right.$ at $\left.4{ }^{\circ} \mathrm{C}\right)$. Cell pellets were washed twice and resuspended in $0.2 \mathrm{~mL}$ of ice-cold $375 \mathrm{mM}$ D-sorbitol to a final concentration of $5 \times 10^{8}$ cells $/ \mathrm{mL}$ and transferred to a precooled $2 \mathrm{~mm}$ electroporation cuvette. Three micrograms of plasmid and $30 \mu \mathrm{g}$ of salmon sperm DNA were added to the resuspended cells and mixed by gently flicking the cuvettes. The transformation was performed using a Bio-Rad GenePulser II with $11 \mathrm{kV} / \mathrm{cm}$ field strength, $50 \mu \mathrm{F}$ capacitance, and $600 \Omega$ shunt. After transformation, the cells were quickly transferred to $5 \mathrm{~mL}$ of fresh media supplemented with $\mathrm{NB}$ and ammonia for $48 \mathrm{~h}$ recovery under medium light conditions. Subsequently, the cells were pelleted and plated on solid media with NB, ammonia, and respective antibiotics. Colonies were observed between 3 and 6 weeks after the plating.

For the RNP-based transformations, the Cas proteins were desalted at $4{ }^{\circ} \mathrm{C}$ to remove the excess salt present in the Cas protein storage buffer. The desalting was performed by multiple rounds of concentration (Amicon Ultra- $0.5 \mathrm{~mL}$, Ultracel-30 $\mathrm{K})$ and resuspension of the Cas protein in $10 \mathrm{mM}$ HEPES and $12.5 \%$ glycerol solution. Subsequently, the RNPs were assembled by mixing an equimolar amount $(10 \mu \mathrm{M})$ of Cas protein and the crRNA (obtained from IDT) at $37^{\circ} \mathrm{C}$ for $15 \mathrm{~min}$ in an Eppendorf tube. From the assembled RNP complex, $30 \mu \mathrm{L}$ of the sample was used for each transformation.

N. oceanica Colony PCR. N. oceanica colonies were resuspended in $20 \mu \mathrm{L}$ of dilution buffer (Plant Direct PCR Master Mix, Thermo Scientific). PCR was performed using $2 \times$ Q5 PCR Master Mix (NEB) with $2 \mu \mathrm{L}$ of the microalgal suspension per reaction.

Fluorescence-Activated Cell Sorting of Transformants. After transformation, NR mutants expressing tdTomato were recovered for 2 days in $5 \mathrm{~mL}$ of ASW-NB and ammonia under medium light conditions. Cells were transferred to $50 \mathrm{~mL}$ of media and incubated for 3 more days. Subsequently, nontdTomato-expressing cells were sorted using a Sony SH800S cell sorter (Sony Biotechnology Inc.). Cultures were diluted to an $\mathrm{OD}_{750}$ of 0.2 , analyzed at sample pressure 4 , and singlet events with suitable chlorophyll a content were selected, as described previously. ${ }^{64}$ The tdTomato fluorescence of events falling into the "Alive" gate was analyzed in the FL2-A channel at $585 \pm 15$ $\mathrm{nm}$ using a detector gain of $45 \%$ (Figure S1). Cells with an FL2A signal of 10-250 (gate "LowRed") were sorted into ASW media, plated on solid media, and grown for 2 weeks.

Fluorescence and Luminescence Assay. The Nlux activity was screened by resuspending $10 \mu \mathrm{L}$ of an $N$. oceanica colony in $100 \mu \mathrm{L}$ of ASW media in a 96-well F-bottom white Lumitrac plate (Greiner). The Nano-Glo substrate (Promega) was diluted 10000 times in ASW media and $100 \mu \mathrm{L}$ was added to the resuspended culture. Luminescence was measured with the following specifications on a BioTek Synergy Mx plate reader; delay: $100 \mathrm{~ms}$, read height: $1 \mathrm{~mm}$, gain: 135, and integration time: $30 \mathrm{~ms}$.

The fluorescence measurement was performed using $150 \mu \mathrm{L}$ of exponentially grown cultures in a 96-well F-bottom dark Lumitrac plate (Greiner). The tdTomato fluorescence was measured with the following settings: endpoint, excitation: 555/ 9.0, emission: $605 / 20.0$, gain: 150 , and read height: $8 \mathrm{~mm}$. The fluorescence and luminescence measurements were obtained using the plate reader Synergy Mx from Biotek.

RNA Isolation. RNA isolation was performed by trizolphenol-chloroform extraction. Microalgal cultures were grown to the exponential phase and $25 \mathrm{~mL}$ of culture was harvested by centrifugation $\left(2000 \mathrm{~g}, 15 \mathrm{~min}, 4^{\circ} \mathrm{C}\right)$. The supernatants were removed immediately, and pellets were resuspended in 0.5-1 $\mathrm{mL}$ TriZol (TRI Reagent from Sigma Life Science). Samples were vortexed and incubated at room temperature for $5 \mathrm{~min}$. Two hundred microliters of chloroform was added and the samples were mixed by inverting tubes 5-10 times. Again, the samples were incubated for $5 \mathrm{~min}$ at room temperature and centrifuged at $12000 \mathrm{~g}$ for $10 \mathrm{~min}$. The upper phase was collected in a new tube and $20 \mu \mathrm{L}$ of $3 \mathrm{M}$ sodium acetate and an equal volume of ice-cold isopropanol were added. The samples were vortexed for $10 \mathrm{~s}$ and centrifuged at maximum speed for 10 min to precipitate the DNA. The top phase was gently removed and centrifuged again for $2 \mathrm{~min}$ to remove all of the liquid phase. The pellet obtained was incubated for another $3 \mathrm{~min}$ at room temperature. Subsequently, the pellet was resuspended in $5 \mu \mathrm{L}$ of DNase I (NEB), $10 \mu \mathrm{L}$ of DNase I reaction buffer (NEB), and $85 \mu \mathrm{L}$ of milliQ water. The samples were incubated at $37^{\circ} \mathrm{C}$ for $15 \mathrm{~min}$. Ten microliter of $3 \mathrm{M}$ sodium acetate was added to the samples and $1 \mathrm{~mL}$ of $-20{ }^{\circ} \mathrm{C}$ ethanol was added to precipitate the RNA. The mixture was vortexed and centrifuged for $5 \mathrm{~min}$ at the maximum speed. Washing was repeated with $300 \mu \mathrm{L}$ of $70 \%$ ethanol. After completely removing the supernatant and drying the tube at room temperature, the pellets were resuspended in $40 \mu \mathrm{L}$ of RNase free milliQ water. The samples were confirmed to be free of DNA contamination by performing PCR specific to the housekeeping gene elongation factor 1 (EEF1A2) ( $N$. oceanica IMET1_NO22G01440). DNase treatment and RNA precipitation were repeated when necessary.

Transcript Quantification by RT-qPCR. cDNA was generated from isolated RNA using SuperScript III Reverse Transcriptase (Invitrogen) according to the manufacturer's instructions, using $500 \mathrm{ng}$ of RNA as the template and genespecific primers complementary toward the $\mathrm{Td}$ tomato and EEF1A2 CDS (Td Tomato: BG23326/BG23327, BG23328/ BG23329, and BG23330/BG23331; EEF1A2: BG23365/ BG23366, BG23367/BG23368). qPCR was performed using the iQ SYBR Green Supermix (Bio-Rad) in a Bio-Rad CFX96 real-time PCR system. One microliter of undiluted cDNA was 
used as the template for qPCR. Three sets of primers were used, amplifying different regions of approximately 250 nucleotides length of the tdTomato gene. For the control, two sets of primers were used, amplifying different regions of approximately 250 nucleotides length from the housekeeping gene elongation factor 1 . The relative repression in the transcript levels was analyzed by the $-\Delta \Delta C_{t}$ method.

\section{ASSOCIATED CONTENT}

\section{S1 Supporting Information}

The Supporting Information is available free of charge at https://pubs.acs.org/doi/10.1021/acssynbio.1c00329.

Figures showing the gating channel used for isolating the fluorescence less colonies via FACS, the graphical representation of Nlux assay activity using various Cas12a gene sequences, details about codon frequencies and the rare codon calculator figures of multiple Cas12a gene sequences used in the study, figures showing various indels observed in the study and loss of large fragments during multiplexed editing; additionally, the fluorescence levels of CRISPRi without the KRAB domains using both Cas9 and Cas12a are depicted; and finally, the details of plasmid creation and GenBank file of all of the major plasmids mentioned in the study (PDF)

Sequences of various genes, primers, and guide RNAs used in the study and the Addgene IDs of plasmids mentioned in the study (XLSX)

\section{AUTHOR INFORMATION}

\section{Corresponding Authors}

Mihris Ibnu Saleem Naduthodi - Laboratory of Microbiology, Wageningen University, Wageningen 6708 WE, The Netherlands; Bioprocess Engineering, Wageningen University, Wageningen 6708 PB, The Netherlands; 10 orcid.org/00000001-9309-3530; Email: mihris.naduthodi@wur.nl

John van der Oost - Laboratory of Microbiology, Wageningen University, Wageningen 6708 WE, The Netherlands;

Email: john.vanderoost@wur.nl

\section{Authors}

Christian S̈̈dfeld - Bioprocess Engineering, Wageningen University, Wageningen 6708 PB, The Netherlands

Emmanouil Klimis Avitzigiannis - Laboratory of Microbiology, Wageningen University, Wageningen 6708 WE, The Netherlands

Nicola Trevisan - Bioprocess Engineering, Wageningen University, Wageningen 6708 PB, The Netherlands

Eduard van Lith - Laboratory of Microbiology, Wageningen University, Wageningen 6708 WE, The Netherlands

Javier Alcaide Sancho - Laboratory of Microbiology, Wageningen University, Wageningen 6708 WE, The Netherlands

Sarah D'Adamo - Bioprocess Engineering, Wageningen University, Wageningen 6708 PB, The Netherlands

Maria Barbosa - Bioprocess Engineering, Wageningen University, Wageningen 6708 PB, The Netherlands

Complete contact information is available at:

https://pubs.acs.org/10.1021/acssynbio.1c00329

\section{Author Contributions}

M.I.S.N. defined the research question, proposed and developed the methodologies for the experiments, performed a major part of the experiments, and analyzed all of the data generated in the study. C.S., E.K.A., N.T., E.v.L., and J.A.S. performed part of the experiments. S.D.A. and M.B. provided feedback on manuscripts and methodologies. J.v.d.O. provided feedback on formulation of research questions, methodologies and reviewed the manuscript.

Notes

The authors declare no competing financial interest.

\section{ACKNOWLEDGMENTS}

The project is funded by the project "Microalgae as a Green Source from Nutritional Ingredients for Food/Feed and Ingredients for Cosmetics by Cost-Effective New Technologies" (MAGNIFICENT), funded by the Bio-based Industries Joint Technology Initiative under the EU Horizon 2020 Research and Innovation Program (project 745754).

\section{REFERENCES}

(1) Goncalves, E. C.; Wilkie, A. C.; Kirst, M.; Rathinasabapathi, B. Metabolic regulation of triacylglycerol accumulation in the green algae: identification of potential targets for engineering to improve oil yield. Plant Biotechnol. J. 2016, 14, 1649-1660.

(2) Hu, Q.; Sommerfeld, M.; Jarvis, E.; Ghirardi, M.; Posewitz, M.; Seibert, M.; Darzins, A. Microalgal triacylglycerols as feedstocks for biofuel production: perspectives and advances. Plant J. 2008, 54, 621639.

(3) Vasudevan, V.; Stratton, R. W.; Pearlson, M. N.; Jersey, G. R.; Beyene, A. G.; Weissman, J. C.; Rubino, M.; Hileman, J. I. Environmental performance of algal biofuel technology options. Environ. Sci. Technol. 2012, 46, 2451-2459.

(4) Wijffels, R. H.; Barbosa, M. J. An outlook on microalgal biofuels. Science 2010, 329, 796-799.

(5) Chisti, Y. Biodiesel from microalgae. Biotechnol. Adv. 2007, 25, 294-306.

(6) Chisti, Y. Biodiesel from microalgae beats bioethanol. Trends Biotechnol. 2008, 26, 126-131.

(7) Edmundson, S. J.; Wilkie, A. C. Landfill leachate-a water and nutrient resource for algae-based biofuels. Environ. Technol. 2013, 34, $1849-1857$

(8) Farooq, W.; Suh, W. I.; Park, M. S.; Yang, J.-W. Water use and its recycling in microalgae cultivation for biofuel application. Bioresour. Technol. 2015, 184, 73-81.

(9) Costa, J. A. V.; De Morais, M. G. The role of biochemical engineering in the production of biofuels from microalgae. Bioresour. Technol. 2011, 102, 2-9.

(10) Smith, V. H.; Sturm, B. S.; Denoyelles, F. J.; Billings, S. A. The ecology of algal biodiesel production. Trends Ecol. Evol. 2010, 25, 301309.

(11) Rodolfi, L.; Chini Zittelli, G.; Bassi, N.; Padovani, G.; Biondi, N.; Bonini, G.; Tredici, M. R. Microalgae for oil: Strain selection, induction of lipid synthesis and outdoor mass cultivation in a low-cost photobioreactor. Biotechnol. Bioeng. 2009, 102, 100-112.

(12) Sukenik, A.; Beardall, J.; Kromkamp, J. C.; Kopecký, J.; Masojídek, J.; van Bergeijk, S.; Gabai, S.; Shaham, E.; Yamshon, A. Photosynthetic performance of outdoor Nannochloropsis mass cultures under a wide range of environmental conditions. Aquat. Microb. Ecol. 2009, 56, 297-308.

(13) Wang, Q.; Lu, Y.; Xin, Y.; Wei, L.; Huang, S.; Xu, J. Genome editing of model oleaginous microalgae Nannochloropsis spp. by CRISPR/Cas9. Plant J. 2016, 88, 1071-1081.

(14) Ajjawi, I.; Verruto, J.; Aqui, M.; Soriaga, L. B.; Coppersmith, J.; Kwok, K.; Peach, L.; Orchard, E.; Kalb, R.; Xu, W.; et al. Lipid production in Nannochloropsis gaditana is doubled by decreasing expression of a single transcriptional regulator. Nat. Biotechnol. 2017, $35,647-652$.

(15) Verruto, J.; Francis, K.; Wang, Y.; Low, M. C.; Greiner, J.; Tacke, S.; Kuzminov, F.; Lambert, W.; McCarren, J.; Ajjawi, I.; et al. 
Unrestrained markerless trait stacking in Nannochloropsis gaditana through combined genome editing and marker recycling technologies. Proc. Natl. Acad. Sci. U.S.A. 2018, 115, E7015-E7022.

(16) Poliner, E.; Takeuchi, T.; Du, Z.-Y.; Benning, C.; Farré, E. M. Nontransgenic marker-free gene disruption by an episomal CRISPR system in the oleaginous microalga, Nannochloropsis oceanica CCMP1779. ACS Synth. Biol. 2018, 7, 962-968.

(17) Naduthodi, M. I. S.; Mohanraju, P.; Südfeld, C.; D’Adamo, S.; Barbosa, M. J.; Van Der Oost, J. CRISPR-Cas ribonucleoprotein mediated homology-directed repair for efficient targeted genome editing in microalgae Nannochloropsis oceanica IMET1. Biotechnol. Biofuels 2019, 12, No. 66.

(18) Wang, Q.; Gong, Y.; He, Y.; Xin, Y.; Lv, N.; Du, X.; Li, Y.; Jeong, B. R.; Xu, J. Genome engineering of Nannochloropsis with hundredkilobase fragment deletions by Cas9 cleavages. Plant J. 2021, 106, $1148-1162$.

(19) Jinek, M.; Chylinski, K.; Fonfara, I.; Hauer, M.; Doudna, J. A.; Charpentier, E. A programmable dual-RNA-guided DNA endonuclease in adaptive bacterial immunity. Science 2012, 337, 816-821.

(20) Ran, F. A.; Hsu, P. D.; Wright, J.; Agarwala, V.; Scott, D. A.; Zhang, F. Genome engineering using the CRISPR-Cas9 system. Nat. Protoc. 2013, 8, 2281-2308.

(21) Cong, L.; Ran, F. A.; Cox, D.; Lin, S.; Barretto, R.; Habib, N.; Hsu, P. D.; Wu, X.; Jiang, W.; Marraffini, L. A.; et al. Multiplex genome engineering using CRISPR/Cas systems. Science 2013, 339, 819-823.

(22) Kilian, O.; Benemann, C. S.; Niyogi, K. K.; Vick, B. Highefficiency homologous recombination in the oil-producing alga Nannochloropsis sp. Proc. Natl. Acad. Sci. U.S.A. 2011, 108, 2126521269.

(23) Poliner, E.; Clark, E.; Cummings, C.; Benning, C.; Farre, E. M. A high-capacity gene stacking toolkit for the oleaginous microalga, Nannochloropsis oceanica CCMP1779. Algal Res. 2020, 45, No. 101664.

(24) Zetsche, B.; Gootenberg, J. S.; Abudayyeh, O. O.; Slaymaker, I. M.; Makarova, K. S.; Essletzbichler, P.; Volz, S. E.; Joung, J.; Van Der Oost, J.; Regev, A.; et al. Cpf1 is a single RNA-guided endonuclease of a class 2 CRISPR-Cas system. Cell 2015, 163, 759-771.

(25) Zetsche, B.; Heidenreich, M.; Mohanraju, P.; Fedorova, I.; Kneppers, J.; DeGennaro, E. M.; Winblad, N.; Choudhury, S. R.; Abudayyeh, O. O.; Gootenberg, J. S.; et al. Multiplex gene editing by CRISPR-Cpf1 using a single crRNA array. Nat. Biotechnol. 2017, 35, $31-34$.

(26) Shaner, N. C.; Campbell, R. E.; Steinbach, P. A.; Giepmans, B. N.; Palmer, A. E.; Tsien, R. Y. Improved monomeric red, orange and yellow fluorescent proteins derived from Discosoma sp. red fluorescent protein. Nat. Biotechnol. 2004, 22, 1567-1572.

(27) Poliner, E.; Pulman, J. A.; Zienkiewicz, K.; Childs, K.; Benning, C.; Farré, E. M. A toolkit for Nannochloropsis oceanica CCMP 1779 enables gene stacking and genetic engineering of the eicosapentaenoic acid pathway for enhanced long-chain polyunsaturated fatty acid production. Plant Biotechnol. J. 2018, 16, 298-309.

(28) Jiang, W.; Brueggeman, A. J.; Horken, K. M.; Plucinak, T. M.; Weeks, D. P. Successful transient expression of Cas9 and single guide RNA genes in Chlamydomonas reinhardtii. Eukaryotic Cell 2014, 13, $1465-1469$.

(29) Naduthodi, M. I. S.; Barbosa, M. J.; van der Oost, J. Progress of CRISPR-Cas based genome editing in photosynthetic microbes. Biotechnol. J. 2018, 13, No. 1700591.

(30) Zhang, Y.-T.; Jiang, J.-Y.; Shi, T.-Q.; Sun, X.-M.; Zhao, Q.-Y.; Huang, H.; Ren, L.-J. Application of the CRISPR/Cas system for genome editing in microalgae. Appl. Microbiol. Biotechnol. 2019, 103, 3239-3248.

(31) Stukenberg, D.; Zauner, S.; Dell'Aquila, G.; Maier, U. G. Optimizing CRISPR/Cas9 for the diatom Phaeodactylum tricornutum. Front. Plant Sci. 2018, 9, No. 740.

(32) Jiang, W. Z.; Weeks, D. P. A gene-within-a-gene Cas9/sgRNA hybrid construct enables gene editing and gene replacement strategies in Chlamydomonas reinhardtii. Algal Res. 2017, 26, 474-480.

(33) Jiang, W.; Zhou, H.; Bi, H.; Fromm, M.; Yang, B.; Weeks, D. P. Demonstration of CRISPR/Cas9/sgRNA-mediated targeted gene modification in Arabidopsis, tobacco, sorghum and rice. Nucleic Acids Res. 2013, 41, No. e188.

(34) Shin, S.-E.; Lim, J.-M.; Koh, H. G.; Kim, E. K.; Kang, N. K.; Jeon, S.; Kwon, S.; Shin, W.-S.; Lee, B.; Hwangbo, K.; et al. CRISPR/Cas9induced knockout and knock-in mutations in Chlamydomonas reinhardtii. Sci. Rep. 2016, 6, No. 27810.

(35) Baek, K.; Yu, J.; Jeong, J.; Sim, S. J.; Bae, S.; Jin, E. Photoautotrophic production of macular pigment in a Chlamydomonas reinhardtii strain generated by using DNA-free CRISPR-Cas9 RNPmediated mutagenesis. Biotechnol. Bioeng. 2018, 115, 719-728.

(36) Baek, K.; Kim, D. H.; Jeong, J.; Sim, S. J.; Melis, A.; Kim, J.-S.; Jin, E.; Bae, S. DNA-free two-gene knockout in Chlamydomonas reinhardtii via CRISPR-Cas9 ribonucleoproteins. Sci. Rep. 2016, 6, No. 30620.

(37) Chang, K. S.; Kim, J.; Park, H.; Hong, S.-J.; Lee, C.-G.; Jin, E. Enhanced lipid productivity in AGP knockout marine microalga Tetraselmis sp. using a DNA-free CRISPR-Cas9 RNP method. Bioresour. Technol. 2020, 303, No. 122932.

(38) Zhang, D.; Hussain, A.; Manghwar, H.; Xie, K.; Xie, S.; Zhao, S.; Larkin, R. M.; Qing, P.; Jin, S.; Ding, F. Genome editing with the CRISPR-Cas system: an art, ethics and global regulatory perspective. Plant Biotechnol. J. 2020, 18, 1651-1669.

(39) Kim, D.; Kim, J.; Hur, J. K.; Been, K. W.; Yoon, S.-h.; Kim, J.-S. Genome-wide analysis reveals specificities of Cpf1 endonucleases in human cells. Nat. Biotechnol. 2016, 34, 863-868.

(40) Claassens, N. J.; Siliakus, M. F.; Spaans, S. K.; Creutzburg, S. C.; Nijsse, B.; Schaap, P. J.; Quax, T. E.; Van Der Oost, J. Improving heterologous membrane protein production in Escherichia coli by combining transcriptional tuning and codon usage algorithms. PLoS One 2017, 12, No. e0184355.

(41) Hall, M. P.; Unch, J.; Binkowski, B. F.; Valley, M. P.; Butler, B. L.; Wood, M. G.; Otto, P.; Zimmerman, K.; Vidugiris, G.; Machleidt, T.; et al. Engineered luciferase reporter from a deep sea shrimp utilizing a novel imidazopyrazinone substrate. ACS Chem. Biol. 2012, 7, 18481857.

(42) Clarke, T. F., IV; Clark, P. L. Rare codons cluster. PLoS One 2008, 3, No. e3412.

(43) Zhang, W.-W.; Matlashewski, G. CRISPR-Cas9-mediated genome editing in Leishmania donovani. mBio 2015, 6, No. e00861-15.

(44) Weninger, A.; Hatzl, A.-M.; Schmid, C.; Vogl, T.; Glieder, A. Combinatorial optimization of CRISPR/Cas9 expression enables precision genome engineering in the methylotrophic yeast Pichia pastoris. J. Biotechnol. 2016, 235, 139-149.

(45) Adiego-Pérez, B.; Randazzo, P.; Daran, J. M.; Verwaal, R.; Roubos, J. A.; Daran-Lapujade, P.; Van Der Oost, J. Multiplex genome editing of microorganisms using CRISPR-Cas. FEMS Microbiol. Lett. 2019, 366, No. fnz086.

(46) Liao, C.; Ttofali, F.; Slotkowski, R. A.; Denny, S. R.; Cecil, T. D.; Leenay, R. T.; Keung, A. J.; Beisel, C. L. Modular one-pot assembly of CRISPR arrays enables library generation and reveals factors influencing crRNA biogenesis. Nat. Commun. 2019, 10, No. 2948.

(47) Kurita, T.; Moroi, K.; Iwai, M.; Okazaki, K.; Shimizu, S.; Nomura, S.; Saito, F.; Maeda, S.; Takami, A.; Sakamoto, A.; et al. Efficient and multiplexable genome editing using Platinum TALENs in oleaginous microalga, Nannochloropsis oceanica NIES-2145. Genes Cells 2020, 25, 695-702.

(48) Ciurkot, K.; Gorochowski, T. E.; Roubos, J. A.; Verwaal, R. Efficient multiplexed gene regulation in Saccharomyces cerevisiae using dCas12a. Nucleic Acids Res. 2021, 49, 7775-7790.

(49) Campa, C. C.; Weisbach, N. R.; Santinha, A. J.; Incarnato, D.; Platt, R. J. Multiplexed genome engineering by Cas12a and CRISPR arrays encoded on single transcripts. Nat. Methods 2019, 16, 887-893.

(50) Larson, M. H.; Gilbert, L. A.; Wang, X.; Lim, W. A.; Weissman, J. S.; Qi, L. S. CRISPR interference (CRISPRi) for sequence-specific control of gene expression. Nat. Protoc. 2013, 8, 2180-2196.

(51) Yao, L.; Cengic, I.; Anfelt, J.; Hudson, E. P. Multiple gene repression in cyanobacteria using CRISPRi. ACS Synth. Biol. 2016, 5, 207-212.

(52) Huang, C.-H.; Shen, C. R.; Li, H.; Sung, L.-Y.; Wu, M.-Y.; Hu, Y.C. CRISPR interference (CRISPRi) for gene regulation and succinate 
production in cyanobacterium S. elongatus PCC 7942. Microb. Cell Fact. 2016, 15, No. 196.

(53) Kao, P.-H.; Ng, I.-S. CRISPRi mediated phosphoenolpyruvate carboxylase regulation to enhance the production of lipid in Chlamydomonas reinhardtii. Bioresour. Technol. 2017, 245, 1527-1537.

(54) Zhang, X.; Wang, J.; Cheng, Q.; Zheng, X.; Zhao, G.; Wang, J. Multiplex gene regulation by CRISPR-ddCpf1. Cell Discovery 2017, 3, No. 17018.

(55) Choi, S. Y.; Woo, H. M. CRISPRi-dCas12a: A dCas12a-mediated CRISPR interference for repression of multiple genes and metabolic engineering in cyanobacteria. ACS Synth. Biol. 2020, 9, 2351-2361.

(56) Gilbert, L. A.; Larson, M. H.; Morsut, L.; Liu, Z.; Brar, G. A.; Torres, S. E.; Stern-Ginossar, N.; Brandman, O.; Whitehead, E. H.; Doudna, J. A.; et al. CRISPR-mediated modular RNA-guided regulation of transcription in eukaryotes. Cell 2013, 154, 442-451.

(57) Gilbert, L. A.; Horlbeck, M. A.; Adamson, B.; Villalta, J. E.; Chen, Y.; Whitehead, E. H.; Guimaraes, C.; Panning, B.; Ploegh, H. L.; Bassik, M. C.; et al. Genome-scale CRISPR-mediated control of gene repression and activation. Cell 2014, 159, 647-661.

(58) Thakore, P. I.; Black, J. B.; Hilton, I. B.; Gersbach, C. A. Editing the epigenome: technologies for programmable transcription and epigenetic modulation. Nat. Methods 2016, 13, 127-137.

(59) Konermann, S.; Brigham, M. D.; Trevino, A. E.; Hsu, P. D.; Heidenreich, M.; Cong, L.; Platt, R. J.; Scott, D. A.; Church, G. M.; Zhang, F. Optical control of mammalian endogenous transcription and epigenetic states. Nature 2013, 500, 472-476.

(60) Yeo, N. C.; Chavez, A.; Lance-Byrne, A.; Chan, Y.; Menn, D.; Milanova, D.; Kuo, C.-C.; Guo, X.; Sharma, S.; Tung, A.; et al. An enhanced CRISPR repressor for targeted mammalian gene regulation. Nat. Methods 2018, 15, 611-616.

(61) Bikard, D.; Jiang, W.; Samai, P.; Hochschild, A.; Zhang, F.; Marraffini, L. A. Programmable repression and activation of bacterial gene expression using an engineered CRISPR-Cas system. Nucleic Acids Res. 2013, 41, 7429-7437.

(62) Naduthodi, M. I. S.; Claassens, N. J.; D’Adamo, S.; van der Oost, J.; Barbosa, M. J. Synthetic Biology Approaches To Enhance Microalgal Productivity. Trends Biotechnol. 2021, 39, 1019-1036.

(63) Vieler, A.; Wu, G.; Tsai, C.-H.; Bullard, B.; Cornish, A. J.; Harvey, C.; Reca, I.-B.; Thornburg, C.; Achawanantakun, R.; Buehl, C. J.; et al. Genome, functional gene annotation, and nuclear transformation of the heterokont oleaginous alga Nannochloropsis oceanica CCMP1779. PLoS Genet. 2012, 8, No. e1003064.

(64) Südfeld, C.; Hubáček, M.; D’Adamo, S.; Wijffels, R. H.; Barbosa, M. J. Optimization of high-throughput lipid screening of the microalga Nannochloropsis oceanica using BODIPY 505/515. Algal Res. 2021, 53, No. 102138. 